\title{
Random Walk Simulation of Fibroblast Cells through RyTrack and Lab VIEW Software Applications
}

\author{
Clare Maristela V. Galon
}

\author{
Department of Chemistry and Physics, College of Arts and Sciences, Cebu Normal University,
} Osmena Boulevard, Cebu City 6000, Cebu, Philippines; galoncm@cnu.edu.ph

\begin{abstract}
Objectives: In this study, the theoretical results of the trajectories of a particle in Brownian motion are presented through simulation using LabVIEW. Methods/Statistical Analysis: Particle-tracking methods, which probe local mechanical properties has been employed. For a 2.0-micron polystyrene bead attached to the walls of fibroblast cells, its movements are tracked through time using video microscopy in which Rytrack software geared with algorithms is used to extract the trajectories of the particles. The time-lag dependence Mean Square Displacement (MSD) of each fibroblast cell is computed from the time-dependent coordinates of that cell. Findings: From the experiment and through simulations of random walk, it is found that as time increases, MSD also increases. It reveals the viscoelastic nature of the microenvironment in the immediate vicinity of each fibroblast cell. From the computed MSDs, the fibroblast cell possesses both having liquidlike and solid-like characteristics. The MSD graph gives characteristic curve that elucidates the nature of fibroblast cell in the system. The analyzed diffusion is of isotropic nature depicted by the linear correlation and the tracers are having sub-diffusive mean trajectories. The mechanical properties of the cytoplasm of fibroblast cells are spatially variable and depend on timescales. Application/Improvement: This study gives characteristic analysis on the mechanical properties of fibroblast cells which are essential for proper function and health.
\end{abstract}

Keywords: Biophysics, Fibroblast Cells, LabVIEW, Random Walk, Rytrack, Simulation

\section{Introduction}

The properties of a biological material include its composition and microscopic structure and the relationship between its structure and its mechanical properties. For living cells, the motor-driven internal motion also significantly impacts the properties, even independently of any remodeling of the cell structure that can occur ${ }^{1}$. These materials' properties dictate the passive mechanical response of the material to an applied force. The mechanical properties of cells and tissues are essential for their function and health and affect how cells actively respond to mechanical force in important biological processes, ranging from motility to differentiation and morphogenesis. The mechanical properties of bulk tissues can be determined by traditional rheological techniques that measure the force required to stretch, compress, or shear macroscopic tissue ${ }^{1}$ Ayala et.al (2016) for example, developed and applied optical tweezers to investigate the rheological behavior of fibroblasts. However, individual cells are too small to be measured by such methods and have highly heterogeneous structures; thus, techniques are required that can probe soft materials at the micrometer scale Gail et.al (1970) used time-lapse cinematography to investigate the motion of mouse fibroblasts in tissue culture and confirmed theoretically and experimentally that a random walk model could describe the motion of fibroblasts in tissue culture. This paper presented microrheological techniques using software applications for optical imaging of micrometer-sized probe on fibroblast cells and for simulating its random walk. It also described the appearance of Brownian motion via direct observation and measurement of the positions of micronsized spherical particles of the cell. 
Cells need to adapt their intracellular physical properties to the physical properties of their extracellular milieu to grow, differentiate, and migrate ${ }^{2}$. Quantifying changes in the mechanical properties of cells, which can be associated with such complex processes, may help to understand biological function ${ }^{3}$. A fibroblast is a type of cell that synthesizes the extracellular matrix and collagen, the structural framework (stroma) for animal tissues, and plays a critical role in wound healing. Fibroblasts are the most common cells of connective tissue in animals. Its main function is to maintain the structural integrity of connective tissues by continuously secreting precursors of the extracellular matrix. It secretes the precursors of all the components of the extracellular matrix, primarily the ground substance and a variety of fibers. The composition of the extracellular matrix determines the physical properties of connective tissues. Fibroblasts exhibit a wide variety of migratory behaviors, such as persistent random walk, contact inhibition of locomotion, and ordered behaviors ${ }^{4}$ Hassan AR et.al (2017) developed a model of cell migration of cells, such as fibroblast cells and found that various collective migratory behaviors may emerge without more restrictive assumptions. The Passucci et al (2019) characterized the motility of mouse fibroblasts on 2D substrates. They have found that it is super-diffusive, which covers distance faster than a random walk but not as fast as a straight walk ${ }^{5}$.

\section{Theory and Methodology}

Microrheology is often performed in complex and heterogeneous systems. It is able to probe a material at a length scale that is below its material structural size. Video microscopy aids microrheology. It tracks particles' movements in time through the motions of the desired number of beads attached on it.

The motion of the particle due to the buffering by the molecules in a gas or liquid is called Brownian motion. The first observation of it is that the particle under the microscope appears to perform a random walk. For a particle executing a random walk, its probability of moving in the positive $\mathrm{x}$-axis ( $\mathrm{y}$-axis) direction in a given step is equal to that for moving in the negative $\mathrm{x}$-direction ( $\mathrm{y}$-direction), and the step lengths are distributed in Gaussian form.

From our analysis of diffusion and random walk, the distribution of step lengths $\Delta x$ in the $x$ direction follows a normalized probability distribution of the form,

$$
P(\Delta x)=\sqrt{\frac{1}{4 \pi D t} \exp \left(\frac{-(\Delta x)^{2}}{4 D t}\right)}
$$

where $D=\frac{k_{B} T}{6 \pi \eta a}$ is the self-diffusion coefficient, $k_{B}$ is Boltzmann's constant, $\eta$ is the viscosity of the solution, $a$ is the particle radius and $t$ is the time between photographs. This states that the probability of finding the particle at $x(t)$ is Gaussian and the width of the Gaussian is time dependent.

In statistical mechanics, the mean-squared displacement is the most common measure of the spatial extent of random motion. It is obtained as an average of all steps corresponding to a single lag time $\Delta t \stackrel{6}{\text { : }}$ :

$$
\begin{aligned}
& <(\Delta r(\Delta t))^{2} \geq \frac{1}{n}\left(\left(\Delta r_{1}(\Delta t)\right)^{2}+\left(\Delta r_{2}(\Delta t)\right)^{2}+\ldots+\left(\Delta r_{i}(\Delta t)\right)^{2}\right) \\
& <(\Delta r(\Delta t))^{2}>=\frac{1}{n} \sum_{i=1}^{n} r_{i}^{2}(\Delta t)
\end{aligned}
$$

We want to limit ourselves to a movement in two dimensions. LabVIEW software was used in the simulation of the random walk of particles undergoing Brownian motion using equation 2 as basis. Images of 2.0-micron polystyrene spheres and fibroblast cells (Figures 1-2) were obtained with bright field microscopy using $100 x$ oil immersion objective (Carl Zeiss Microscopy) with 1.2 N.A. (Numerical Aperture), at a magnification of $(0.101 \pm 0.001) \mu \mathrm{m} / \mathrm{CCD}$ pixel. These images were saved sequentially in Joint Photographic Experts Group (JPEG) format for processing and tracking of probe particles. RyTrack, a front end GUI-based particle tracking interface, is coupled with the microrheology tools and algorithms developed by Crocker, et.al which is the Interactive Data Language (IDL) programming. Analysis is limited to the first 10 seconds due to the diffusing out of the beads from the viewing field through time. 


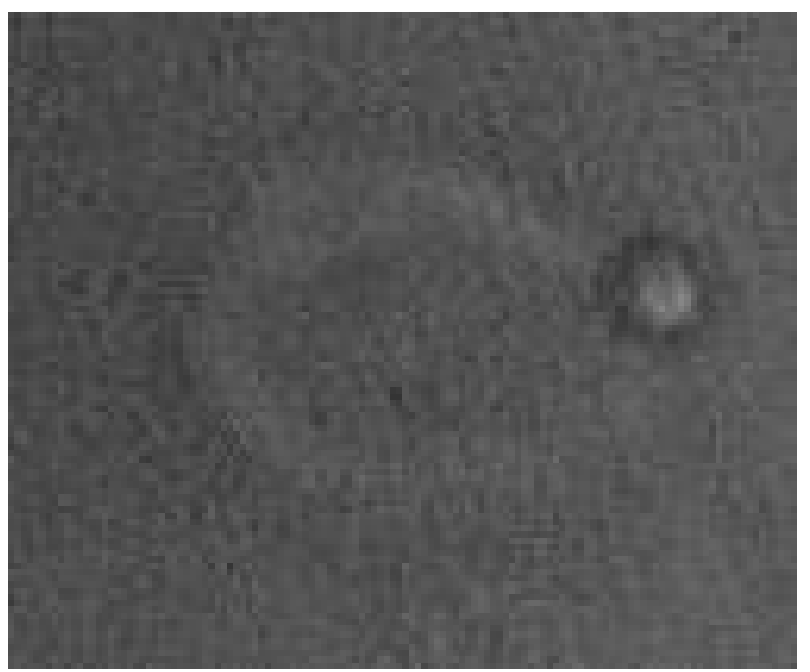

Figure 1. Image of a fibroblast cell captured using ChargeCoupled Device (CCD) with a 2.0 micron size polystyrene bead (sphere) attached on the surface.

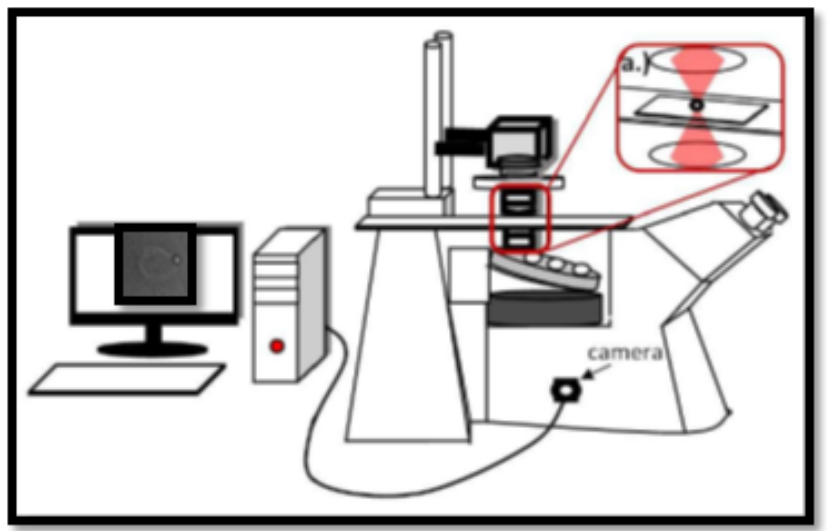

Figure 2. The set-up used in the experiment for the fibroblast cells. It consists of bright field microscopy with oil immersion objective, camera, and computer for the image acquisition. The samples are placed in $(a)$.

\section{Results and Discussion}

The random walk of a fibroblast cell attached with a fibronectin-coated polystyrene bead is shown in the $\mathrm{x}-\mathrm{y}$ graph of Figure 3. This shows the 1000 different positions of the bead captured within 30 seconds. Figure 4 is the random walk simulated using LabVIEW. From the graphs, we can see that our cell is undergoing random motion and some of its movements have the same $\mathrm{x}$ and $\mathrm{y}$ positions through time. A confined random walk and partially confined random walk can be depicted. The MSD plotted to its corresponding step time interval changes depending on the type of random walk (Figure 5). There is a similarity between our simulated results and the real-time movements of our experimented cell. Continuous lines on Figures 3\&4 indicate the displacement corresponding to single steps while dotted lines corresponding to double step during two time intervals.

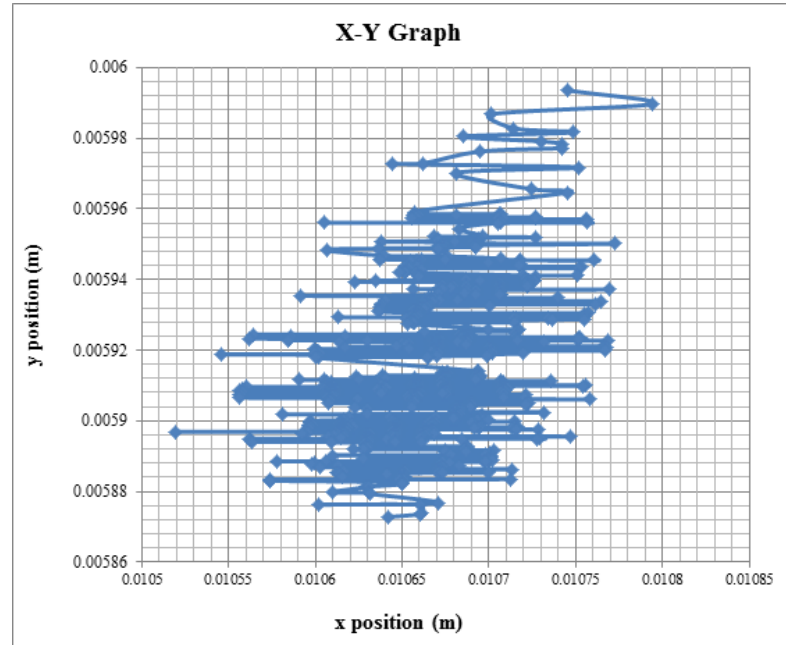

Figure 3. Graph of the random walk of a fibroblast cell through time. Some have moved in the same $\mathrm{x}$ and $\mathrm{y}$ positions.

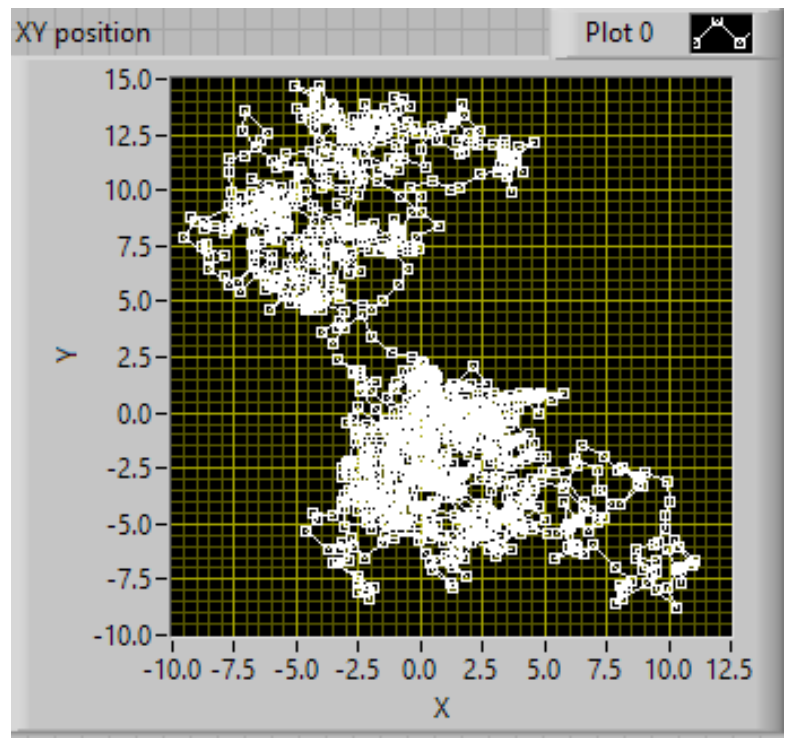

Figure 4. (Front Panel) Simulation of the $\mathrm{X}$ and $\mathrm{Y}$ positions of particles undergoing random motion using the lab VIEW software. 


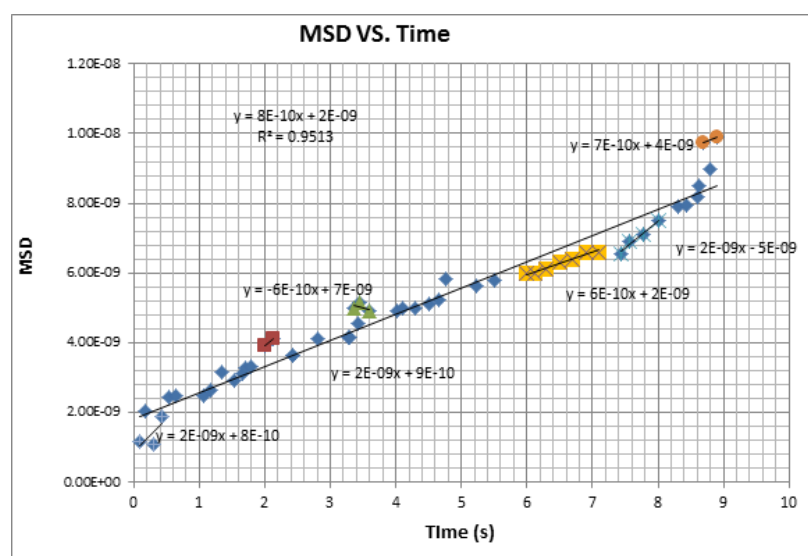

Figure 5a. Graph of MSD versus time of fibroblast cell within 10 secs., fitted with a linear trendline. The slope of the line is equal to the diffusion coefficient.

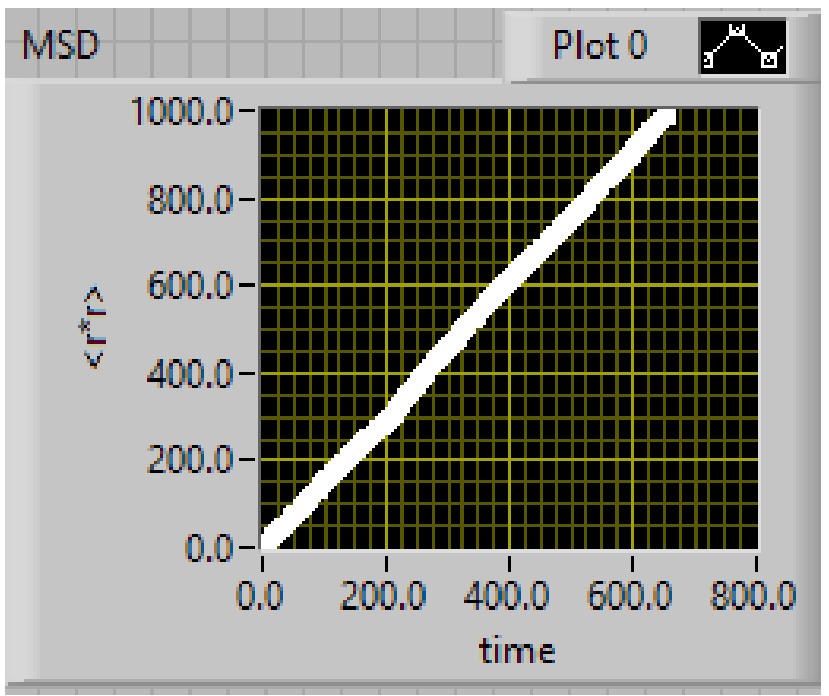

Figure 5b. (Front Panel) Graph of MSD versus time of the random $\mathrm{X}$ and $\mathrm{Y}$ positions as simulated using the LabVIEW software.

Figure 5 shows graphs of the computed MSD versus time of fibroblast cell within 10 seconds (Figure 5a) and the simulated results (Figure 5b). The graphs show a positive increase of MSD. Figure $5 \mathrm{a}$ is fitted to a linear trend line that gives slopes equal to the diffusion coefficient. There are deviations seen with increasing slopes due to the specifically chaotic mechanism which is a long time considered to be a property of living or at least organic matter. This is also due to the rate of diffusion of the cell which is affected by its properties and its surrounding solution. We can also attribute it to cell attachments because it entails a very good contact of our beads to our fibroblast cells for us to track its motion correctly. Other main sources of uncertainty resulting from image-fitting approaches are due to noise and camera exposure ${ }^{7,8}$. Figure 6 shows the time Line Fit Plot from the regression analysis made with $95 \%$ confidence level. The blue diamond plots correspond to our computed MSD using equation 2 above and the red square plots are the predicted MSD which is linear with time. In the case of diffusion restricted to a confined space the MSD naturally does not exceed the diameter of this space, on the other hand one often finds a partially confined random walk in biological matter.

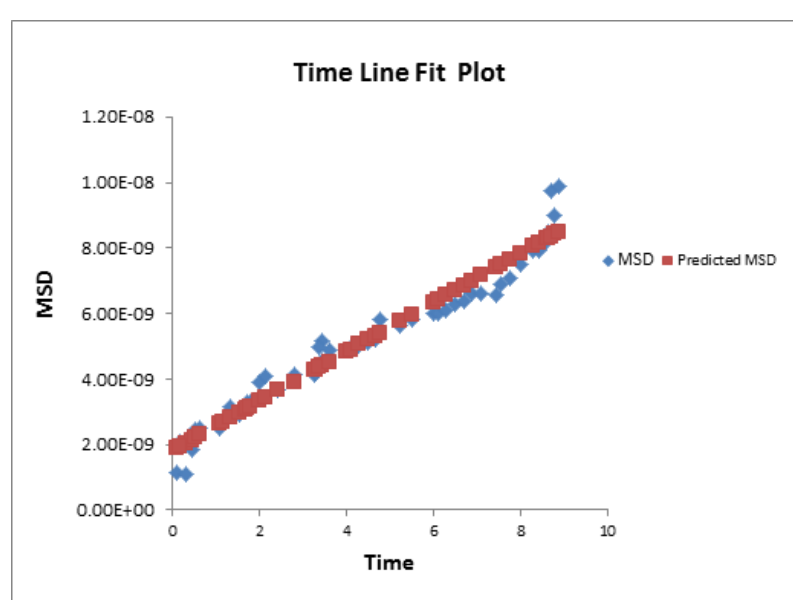

Figure 6. Graph of MSD versus time within 10 secs. and the time Line Fit Plot from the regression analysis made with $95 \%$ confidence level.

\section{Conclusions}

Video microscopy is one of the most efficient tools in studying characteristics of particles undergoing Brownian motion in microrheology. Experimental techniques developed in this study have successfully correlated to the theoretical approach through simulated results using Lab VIEW software in studying the diffusion or random walk of fibroblast cells. The cell possesses viscoelastic properties and the tracers are having sub-diffusive mean trajectories.

\section{Acknowledgements}

The author would like to thank Dr. Rommel G. Bacabac, head of the Medical BioPhysics Group, Department of Physics, USC, for his knowledge and expertise on the topic. 


\section{References}

1. Kasza K, Vader D, Köster S, Wang N, Weitz D. Imaging techniques for measuring the materials properties of cells, Cold Spring Harb. Protoc. 2011; (4). https://doi.org/10.1101/ pdb.top107. PMid:21460060.

2. Wirtz D. Particle-tracking microrheology of living cells: principles and applications, Annual Reviews of Biophysics. 2009; 38:301-26. https://doi.org/10.1146/annurev.biophys.050708.133724. PMid: 19416071.

3. Bacabac R, Mizuno D, Koenderink G. Mechanical Properties of Living Cells: On Mechanosensing and Microgravity, Book Chapter, Cell Mechanochemistry. Biological Systems and Factors Inducing Mechanical Stress, Such As Light, Pressure and Gravity; 2010. p. 23-54.

4. Hassan AR, Biel T, Umulis DM, Kim T. Interplay between the persistent random walk and the contact inhibition of locomotion leads to collective cell behaviors, Bull Math Biology. 2019. https://doi.org/10.1007/s11538-019-005851. PMid: 30788690.
5. Passucci G, Brasch ME, Henderson JH, Zaburdaev V, Manning ML. Identifying the mechanism for super diffusivity in mouse fibroblast motility, PLoS Comput. Biol. 2019; 15(2). https://doi.org/10.1371/journal.pcbi.1006732. PMid: 30763309, PMCid: PMC6392322.

6. Michalet X. Mean square displacement analysis of singleparticle trajectories with localization error: Brownian motion in isotropic medium, Physical Review E. 2010; 82(4). https://doi.org/10.1103/PhysRevE.82.041914. PMid: 21230320 , PMCid: PMC3055791.

7. Gail MH, Boone CW. The locomotion of mouse fibroblasts in tissue culture, Biophysical Journal. 1970; 10. https://doi. org/10.1016/S0006-3495(70)86347-0.

8. Ayala YA. Rheological properties of cells measured by optical tweezers, BMC Biophysics. 2016; 9(5). https://doi. org/10.1186/s13628-016-0031-4. PMid: 27340552, PMCid: PMC4917937. 